\title{
Sero-pravelence of SARS CoV-2 IgM and IgG Antibodies Amongst Blood Donors in Nigeria
}

ljeoma Ifeorah

University of Nigeria Faculty of Health Sciences and Technology

Emmanuel Nna ( $\nabla$ e.nna@themping.org)

Molecular Pathology Institute https://orcid.org/0000-0001-6791-2336

Uchenna Okeke

Nigerian Navy Reference Hospital Calabar

Emo Ivo

Ministry of Defence, Health Implementation Programme

Ojor Ayemoba

Ministry of Defence, Health Implementation Programme

Thairu Yunusa

University of Abuja Teaching Hospital

Ogbeche Ochagu

Headquarters Surgeon General: Defence Medical Services

Ngozi Ugwu

Federal Teaching Hospital Abakaliki: Alex Ekwueme Federal University Teaching Hospital Abakaliki

Henrietta Okafor

UNTH: University of Nigeria Teaching Hospital

Amaka Nnamani

UNTH: University of Nigeria Teaching Hospital

Nneka lloanusi

UNTH: University of Nigeria Teaching Hospital

Chika Onu

The Molecular Pathology Institute Enugu

Ifeoma Okoye

UNTH: University of Nigeria Teaching Hospital

\section{Research}

Keywords: SARS CoV-2, sero-prevalence, COVID-19, immunity, blood donors, IgG, IgM, Nigeria, herd immunity, community transmission

Posted Date: January 21st, 2021 
DOI: https://doi.org/10.21203/rs.3.rs-151037/v1

License: (c) (1) This work is licensed under a Creative Commons Attribution 4.0 International License. Read Full License 


\section{Abstract}

Background: Currently in Nigeria, SARS CoV-2 infection control measures rely solely on RT-PCR testing of cases with high index of suspicion for COVID-19. The sero-prevalence of SARS-CoV-2-specific antibodies in the populations remains unknown. We aimed to determine the sero-prevalence of SARS CoV-2 IgM and IgG in screened blood donors in two selected cities in Nigeria.

Methods: A total of 113 blood samples from screened and accepted blood donors were tested for both SARS CoV-2 IgM and IgG antibodies using NOVATECH ELISA kits from Eurofins Germany.

Results: The age range of donor was 21-48 years. Males were $96 \%$. Of the 113 blood donors $42 \%$ were positive for SARS CoV-2 IgG while $41 \%$ were positive for SARS CoV-2 IgM respectively. Only $11 \%$ and $19 \%$ of blood donors had equivocal test result for IgG and IgM respectively.

Conclusions: Nearly a half of accepted blood donors in Nigeria had been exposed to SARS CoV-2 infection. Although it is not a blood transmissible infection, the blood banks must take adequate preventive measures to prevent spread amongst the bank staff and potential donors. It also implies a wider spread of the infection in Nigeria.

Trial Registration: The study does not require registration.

\section{Background}

The emergence of Severe acute respiratory coronavirus 2 (SARS-CoV-2) and subsequent outbreak of COVID-19, is a global health challenge which has culminated in significant morbidity and mortality [1]. The virus can be transmitted in humans by direct or indirect contact with respiratory droplets of an infected person [2]. Although the viral RNA has been detected in stool, whole blood and urine of COVID-19 patients, transmission via these routes have not yet been documented $[3,4]$. Infection with SARS-CoV-2 often presents with no symptom or a range of mild symptoms, including high temperature, dry cough, muscle pain, anosmia etc, and can progress into severe forms of the illness and possible death especially in the elderly and/or persons living with other comorbidities $[5,6]$. Asymptomatic infections may be a substantial source of transmission and a challenge to infection control measures [7].

The first confirmed case of COVID-19 in sub-Saharan Africa was reported in Lagos, Nigeria on February 28,2020 , and as at 23rd November, 2020, a total of 743,298 RT-PCR tests had been performed in Nigeria, resulting in 66,383 onfirmed cases and 1,167 deaths (https://www.worldometers.info/coronavirus/\#countries ) [8]. Being a novel virus, sero-prevalence in a given population is presumed to be dynamic (negligible at onset and changing over the course of time). To date SARS-CoV-2 surveillance especially in Nigeria has focused primarily on individuals with high index of suspicion of COVID 19 even when it is apparent that there exist community transmissions. Although information on laboratory confirmed cases using RT PCR and deaths is important in monitoring the dynamics of the disease, they are less than ideal in estimating the proportion of infection in the entire 
population [9]. Thus sero-prevalence studies are needed for an enhanced monitoring of the evolution of the outbreak and estimation of the actual infection fatality rate in the population

A quick search on SeroTracker [9]; a custom-built dashboard that systematically follows up on SARS-CoV2 serological studies globally and displays findings on an interactive world map, shows paucity of data from sub-Saharan Africa. The SeroTracker enables easy comparison of estimates between regions and population groups clearly. Sero-surveillance of the populace allows inferences to be drawn on the extent of spread and cumulative incidence of infection in the population. Epidemiological studies focusing on the community will provide relevant data and expand our understanding of the spectrum of the disease.

Currently in Nigeria, given that SARS CoV-2 contact tracing relies solely on RT-PCR, the sero-prevalence of SARS-CoV-2-specific antibodies in different populations remains unclear. Consequently, the full spread of the disease, including the extent of mild or asymptomatic infections that do not require medical attention are not documented. Studies to determine the sero-prevalence SARS-CoV-2-specific antibodies in the community and specific population are paramount. These will provide means of measuring the extent of spread and appraise current control measures put in place by the authorities to curb the transmission of the virus. Additionally, knowledge provided by these studies will be found useful by policy makers in calibrating social interventions.

Although, there is frequently updated information on many aspects of the virus spread in Nigeria (e.g., numbers of patients tested positive for SARS-CoV-2, admitted and discharged from the hospital, mortalities) from the Nigeria Centre for Disease Control (NCDC), in contrast, there is very limited data on the percentage of the population with previous mild or asymptomatic infections. The proportion of the population with past and asymptomatic but active infection can fairly be estimated by testing for total antibody, IgG, IgA and IgM antibodies specific to SARS-CoV-2. These antibodies may confer immunity to repeat infection, although it remains unclear how long these immunities could last [10]

Healthy volunteers drawn from community blood donation units of major hospitals in Nigeria may provide vital sero-prevalence data on Covid-19 infection. Therefore, our study was aimed at seroprevalence of SARS CoV-2 IgM and IgG in apparently healthy blood donors who had been recruited, screned and accepted to donate blood in the blood bank. This group of the population will provide a practical glimpse of the current situation of the virus spread at the community level.

\section{Methods}

Ethical Approval: The study received ethical approval from two Health Research Ethics Committees: from the Federal Capital Territory Health Research Ethics Committee Abuja, Approval Number

FHREC/2020/01/77/11-08-20; and The Molecular Pathology Institute Health Research Ethics Committee Enugu, Approval Number MPIHREC/2020/08/02/0002. The study was carried out in compliance with the WMA declaration of Helsinki as amended in 2013 and the Nigerian National Code of Health Research Ethics 2007. 
Aim of study: The aim of this study was to determine the seroprevalence of SARS CoV-2 IgM and IgG antibodies amongst blood donors in Nigeria.

Study Design: This was a cross sectional study with non-probability sampling.

Sample Size: Sample size was calculated using the Raosoft Sample size Calculator (https://raosoft.com). We used a prevalence of $15 \%$ since the pandemic was still evolving. At $5 \%$ alpha level of significance and $95 \%$ confidence level, a sample size of $\mathbf{1 1 3}$ had $80 \%$ power to determine seroprevalence up to estimated prevalence of $15 \%$.

Sampling: A hundred and thirteen informed consented blood donors were recruited during the month of August 2020 to participate in the study. Specifically, these blood donors were drawn from blood donation centers in the city of Abuja and Enugu located in the north central and south eastern part of Nigeria respectively. Briefly about $5 \mathrm{ml}$ od blood was collected from each participant after having been screened and certified eligible to donate according to National Blood Transfusion Service (NBTS) guidelines. The blood was centrifuged at $6000 \mathrm{rpm}$ for 10 minutes and plasma was harvested. Plasma samples were frozen in minus $20^{\circ} \mathrm{C}$ freezer and later transferred on cold chain to the testing laboratory, Safety Molecular Pathology Laboratory, where samples were frozen at $-86^{\circ} \mathrm{C}$ until required for testing. Storage period was no more than 1 month. All samples were screened for SARS-CoV-2 IgM and IgG antibodies using a semi quantitative Enzyme Linked Immunosorbent Assays (ELISA) test kits (NOVA TECH, Germany).

Enzyme Linked Immunosorbent Assays (ELISA): ELISA kits for SARS CoV-2 IgM and IgG antibodies were obtained from Eurofins, Germany, they were manufactured by NOVA TECH Germany. Both assays were semi-quantitative and had a cut of 11 NTU (Nova Tech Units) for positivity. SARS CoV-2 IgM was tested separately from SARS CoV-2 IgG. Tests were carried out according to the Manufacturer's instructions. Briefly, microwell titre plates pre-coated with specific antigens were selected according to the number of samples to be analyzed and 100 ul of diluted sample or control was added into appropriate well. Each sample was diluted in 1 in 100. Positive, cut-off and negative controls were prediluted ready to use. Wells were covered with a foil and incubated for 1 hour at $37^{\circ} \mathrm{C}$. After incubation, the foil was removed, and the contents of each well was aspirated and 300 ul of 1x Wash Buffer was added. Overflows were avoided. The microwell plates were washed thrice. Plates were tapped on tissue paper to ensure that all moisture was removed and $100 \mathrm{ul}$ Conjugate solution was added into all wells. The wells were incubated for 30 minutes at room temperature. After the incubation, the washing steps were repeated using $300 \mathrm{ul}$ of the $1 \mathrm{x}$ Wash buffer. Then $100 \mathrm{ul}$ of TMB Substrate Solution was added into each well and the wells incubated for 15 minutes at room temperature in the dark. At this point blue colours appeared in the wells due to enzymatic reaction. Then 100 ul of Stop Solution was added into all the wells, with the colours changing to yellow. The absorbance of the wells was read at 450/620 nm using Mindray M96 Microwell Plate Reader. In each run a substrate blank was included, in addition to the positive, negative and cut-off controls. The results were calculated according to the manufacturer's instruction and expressed in Nova 
Tech Units (NTU) as follows: > 11 NTU: Positive for the specific antibody, < 9 NTU: Negative for the specific antibody, 9-11 NTU: Equivocal for the specific antibody.

Assay Characteristics: Assay specific characteristics as stated by the manufacturer, for SARS CoV-2 IgG: the assay diagnostic specificity was $100 \%$ (95\% Cl: $95.82-99.98)$ and diagnostic sensitivity at $>12$ days post symptom onset was $100 \%$. SARS CoV-2 IgM: the assay diagnostic specificity was $100 \%(95 \% \mathrm{Cl}$ : 97.26-100.00) and diagnostic sensitivity at > 12 days post symptom onset was $57.14 \%$ (95\% Cl: $18.84-$ 73.37)).

Data Analysis: Data was analyzed in GraphPad Prism version 9 (www.graphpad.com). Gaussian distribution was tested for using D'Agostino Omnibus Normality test. Based on the outcome of the normality test, non-parametric statistics was used to analyze the data. P value was set at 0.05 . For descriptive statistics, median, $95 \%$ confidence interval of the median and inter-quartile range were reported. For inferential statistics comparing positive, negative and equivocal cases, Kruskal Wallis test and Dunn's multiple comparison were performed.

\section{Results}

Of the 113 blood donors, $96 \%$ were males while $4 \%$ were female. Table 1 shows the demographics of the blood donors. The age range for the blood donors were 21-48 years, median age was 33 years.

Descriptive statistics of SARS CoV-2 IgM and IgG are shown in Table 2. Of the 113 blood donors studied, 48 (42\%; 95\% Cl: 34-52\%) were positive for IgG with a median antibody level of 19 NTU; 46 (41\%; $95 \%$ Cl: 32-50 \%) were positive for IgM level with a median antibody level of 5 NTU. Only 11 (11\%; $95 \%$ Cl: 05 - 17\%) donors were equivocal (indeterminate) for SARS CoV-2 IgG, with a median antibody level of 10 NTU. Only 21 (19\%; 95\% Cl: 12-27\%) donors were equivocal (indeterminate) for SARS CoV-2 IgM, with a median antibody level of 10 NTU. Inferential comparison of antibody levels in positive, negative and equivocal cases are shown in Table 3. Analysed independently, Abuja $(n=60)$ had SARS CoV-2 IgG prevalence of $53 \%$ (95\% Cl: $41-65 \%)$ while Enugu $(n=53)$ had SARS CoV-2 IgG prevalence of $31 \%$ ( $95 \%$ Cl: 19-44 \%). Whilst $45 \%$ of Abuja blood donors were SAR CoV-2 IgM positive (95\% Cl: 33-58\%), but $37 \%$ of Enugu blood donors were SARS CoV-2 IgM positive (95\% Cl: $26-51 \%$ ).

\section{Discussion}

The SARS-CoV-2 infection in most sub-Saharan African nations appeared to have a less fatal clinical outcome in comparison with other nations in many parts of the world ${ }^{11}$. Several reasons have been speculated to be responsible for the 'supposed' milder infections in individuals with COVID 19 in the region [11-13]. As at the time of our cross-sectional study in August 2020, Nigeria's COVID 19 situations had plateaued with the society actively re-engaged. Our serological survey showed IgG and IgM seropositivitives of $42 \%$ and $41 \%$ respectively indicating that a greater proportion of individuals in our study population had been either in an active but asymptomatic infection or a previous exposure to SARS-CoV-2. But RT-PCR based results, from the NCDC reports, had suggested a low rate of COVID 19 
infection in Nigeria. The NCDC reported low rate was expected as the country only tested individuals with only high suspicion index for COVID 19 while leaving out those who were probably infected but asymptomatic. The high rate of seropositivity to SARS-CoV-2 antibodies in our study suggests a wider circulation of the virus at the community level in the two cities that were sampled. By implication, more Nigerians had been infected than officially reported and more were still infected as indicated by the high SARS CoV-IgM sero-prevalence.

Our finding is comparable to rates ranging from $24.4 \%-33.7 \%$ obtained at different points in time among blood donors in Pakistan and France $[14,15]$. Our result however contrasted with the rates of 0.02 $\%-10.9 \%$ from blood donors in other parts of the world including those with worst experiences during the initial phase of the pandemic ${ }^{16-20}$. Differences in health policies and adherence to social interventions by the general population in the various countries may have contributed to the variations in the seroprevalence recorded. Futhermore, the testing strategy in Nigeria which did not include antibody testing of asymptomatic persons might have led to many missed cases that continued to interact with other people in the community thus fueling the transmission of the virus. In addition, adherence to social control measures and other COVID 19 protocols was generally poor in most cities in Nigeria [21]. These might have contributed to the high sero-prevalence reported in this study. This poor adherence could be due to many reasons including but not limited to the living conditions of many urban dwellers in Nigeria that might make it impractical to observe physical distancing guidelines and sheer apathy or ignorance toward measures to limit virus spread.

The high rate of actively infected yet asymptomatic persons as shown by the IgM seropositivity in our study confirms the speculation that most infections in sub-Saharan Africa may be asymptomatic due to a large number of younger poeople in the population [12]. Although blood banking policies in Nigeria allowed for blood donation by individuals within the age range 15-65 years, our study shows the blood donors to be in the range of 21-48 years (young and middle aged). Studies have shown that all ages are susceptible to infection by SARS CoV-2, but older age is a major indicator for symptomatic active cases [22-25]. Significantly, this may have a considerable public health implication as many of these young actively infected individuals who are asymptomatic will most likely infect more people than symptomatic individuals [26]. Therefore, it becomes imperative that peculiarities of Nigerian population and situations be put into consideration while building a country specific predictive model that will provide policymakers with several prediction scenarios, based on different actions they can take to address the pandemic.

In this cross-sectional study, the seroprevalence in Abuja being higher than Enugu is expected as Abuja was one of the epicenters of Nigeria COVID 19 experience during the initial phase of the pandemic. Thus, individuals in Abuja may have been at a greater risk of exposure to the virus than Enugu. Although the point of surveillance was about a month after the ban on inter-city movement of people was lifted in Nigeria, possibility exists that in many months to come such may not be the picture of the epidemics. Therefore, point to point sero-surveillance of the population is needed to monitor trends in the virus transmission to inform appropriate health policies. Although, no association between sex and 
susceptibility to SARS-CoV-2 infection has been reported [24], we could not make any inference on this as over $95 \%$ of our study population were males.

There is still no consensus among the scientific community on the implication of $\lg G$ antibodies to SARSCoV-2 in terms of protection and corelates of such protection (if it exists) [27]. It is therefore difficult to extrapolate if these individuals with previous exposure to the virus are protected from future infection and if so, how long such protection will last. With documented cases [28-31] of reinfection after initial encounter with the virus and in some of the cases with worse symptoms than the initial encounter, one will suggest that the concept of immunity passport or herd immunity be treated with caution until more data on immune correlates of protection are available. There is possibility that reinfections are currently being underestimated as it is biased towards detection of symptomatic cases. Consequently, the extent to which reinfection occur in the general population among asymptomatic cases (who may have recovered from their previous infection albeit unknowingly) is still not clear. Hence, calculated utilization of antibody testing will help shed more light to immune correlates of SARS-CoV-2 infection.

One of the limitations of the study was the predominant number of young to middle-aged men, with fewer women involved. Secondly, our study was limited to events at the point of surveillance. Therefore, more serological studies are still required to uncover the rate of asymptomatic infections in the Nigerian population.

\section{Conclusions}

the high prevalence of IgG and IgM antibodies to SARS-CoV-2 reported in this study showed a wider spread of SARS CoV 2 infection in Nigeria. This highlights the need for more elaborate testing protocol that would include antibody testing of the population for epidemiological purposes, especially to uncover the actual rate of infections in Nigeria.

\section{Abbreviations}

ELISA: Enzyme linked immunosorbent assay

SARS CoV-2: Severe Acute Respiratory Syndrome Coronavirus 2

COVID 19: Coronavirus Disease (2019)

IgM: Immunoglobulin M

IgG: Immunoglobulin G

\section{Declarations}

\section{Ethics approval and Consent to participate:}


Favourable ethical opinions were obtained from two Health Research Ethics Committees. Written informed consent was obtained from each participant.

The Federal Capital Territory Health Research Ethics Committee Abuja, Approval Number FHREC/2020/01/77/11-08-20.

The Molecular Pathology Institute Health Research Ethics Committee Enugu, Approval Number MPIHREC/2020/08/02/0002.

\section{Consent for Publication:}

All authors consented to publication

\section{Availability of data and materials:}

All data sets and materials will be made public unhindered.

\section{Competing Interest:}

None

\section{Funding:}

The study was funded by the Molecular Pathology Institute Enugu

\section{Authors' Contributions:}

EN and II conceived and designed the study, UO, El and OA reviewed the study and sampling method, OA, TY, $\mathrm{OO}$ and NU obtained ethical approval and sampled donors, $\mathrm{HO}, \mathrm{AN}, \mathrm{NI}, \mathrm{CO}$ AND $\mathrm{IO}$ did lab testing, data collection. Early manuscript was drafted by II, EN expanded the manuscript fully, all authors contributed to revising the manuscript, EN and II did statistical analysis of data; all authors reviewed the manuscript.

\section{Acknowledgement:}

We are very grateful to the staff of the Molecular Pathology Institute, Enugu for assisting in the study.

\section{References}

1. Zhou. P., Yang, X, I., Wang, X.G. et al. (2020). A pneumonia outbreak associated with a new coronavirus of probable bat origin. Nature. https://doi.org/10.1038/s41586-020-2012-7.

2. Neerukonda, S. N., \& Katneni, U. (2020). A Review on SARS-CoV-2 Virology, Pathophysiology, Animal Models, and Anti-Viral Interventions. Pathogens (Basel, Switzerland), 9(6), 426. https://doi.org/10.3390/pathogens9060426 
3. Young BE, Ong SWX, Kalimuddin S, Low JG, Tan SY, Loh J, et al. Epidemiologic features and clinical course of patients infected with SARS-CoV-2 in Singapore. JAMA. 2020 Mar 3; doi: 10.1001/jama.2020.3204. [Epub]. [PMC free article] [PubMed] [CrossRef] [Google Scholar] [Ref list]

4. To KK, Tsang OT, Chik-Yan Yip C, Chan KH, Wu TC, Chan JMC, et al. Consistent detection of 2019 novel coronavirus in saliva. Clin Infect Dis. 2020 Feb 12; doi: 10.1093/cid/ciaa149. [Epub]. pii: ciaa149.u

5. Xu Z, Shi L, Wang Y, Zhang J, Huang L, Zhang C, et al. Pathological findings of COVID-19 associated with acute respiratory distress syndrome. Lancet Respir Med. 2020;8(4):420-422. doi: 10.1016/S2213-2600(20)30076-X.

6. Chen N, Zhou M, Dong X, Qu J, Gong F, Han Y, et al . Epidemiological and clinical characteristics of 99 cases of 2019 novel coronavirus pneumonia in Wuhan, China: a descriptive study. Lancet. 2020; 395 (10223): 507 - 13 . 10.1016/S0140-6736(20)30211-

7. Wu and McGoogan Infectious Diseases of Poverty (2020) 9:72https://doi.org/10.1186/ s40249-02000679-2

8. Worldometer. https://www.worldometers.info/coronavirus/\#countries. Assessed on 23rd November 2020.

9. Arora, R. K., Joseph, A., Van Wyk, J., Rocco, S., Atmaja, A., May, E., Buckeridge, D. L. (2020). SeroTracker: a global SARS-CoV-2 seroprevalence dashboard. The Lancet Infectious Diseases. doi:10.1016/s1473-3099(20)30631-9

10. Kirkcaldy RD, King BA, Brooks JT. COVID-19 and Post infection Immunity: Limited Evidence, Many Remaining Questions. JAMA. 2020;323(22):2245-2246. doi:10.1001/jama.2020.7869

11. M. Mbow, B. Lell, S.P. Jochems, B. Cisse, S. Mboup, B.G. Dewals, A. Jaye, A. Dieye, M. Yazdanbakhsh, COVID-19 in Africa: Dampening the storm? Science 369, 624626 (2020). doi:10.1126/science.abd3902pmid: 32764055 Abstract/FREE Full TextGoogle Scholar

12. Diop BZ, Ngom $M$, Pougué Biyong $C$, et al The relatively young and rural population may limit the spread and severity of COVID-19 in Africa: a modelling study BMJ Global Health 2020;5: e002699.

13. Payne C. COVID-19 in Africa. Nat Human Behav. 2020;4:436-437. doi: 10.1038/s41562-020-0870-5. [PubMed] [CrossRef] [Google Scholar

14. Fontanet A, Tondeur L, Madec $Y$ et al. Cluster of COVID-19 in northern France: A retrospective closed cohort study. Medrxiv 2020; 2020.04.18.20071134.

15. Younas, A., Waheed, S., Khawaja, S., Imam, M., Borhany, M., \& Shamsi, T. (2020). Seroprevalence of SARS-CoV-2 antibodies among healthy blood donors in Karachi, Pakistan. Transfusion and Apheresis Science, 102923. doi:10.1016/j.transci.2020.102923

16. Xu, R., Huang, J., Duan, C., Liao, Q., Shan, Z., Wang, M., Rong, X., Li, C., Fu, Y., \& Wang, H. (2020). Low prevalence of antibodies against SARS-CoV-2 among voluntary blood donors in Guangzhou, China. Journal of medical virology, 10.1002/jmv.26445. Advance online publication.

17. Amorim Filho, L., Szwarcwald, C. L., Mateos, S., Leon, A., Medronho, R. A., Veloso, V. G., Lopes, J., Porto, L., Chieppe, A., Werneck, G. L., \& Grupo Hemorio de Pesquisa em Covid-19 (2020). 
Seroprevalence of anti-SARS-CoV-2 among blood donors in Rio de Janeiro, Brazil. Revista de saude publica, 54, 69.

18. Vassallo, R. R., Bravo, M. D., Dumont, L. J., Hazegh, K., \& Kamel, H. (2020). Seroprevalence of Antibodies to SARS-CoV-2 in US Blood Donors. doi:10.1101/2020.09.17.20195131

19. Performance of a point of care test for detecting IgM and IgG antibodies against SARS-CoV-2 and seroprevalence in blood donors and health care workers in Panama. (n.d.). doi:10.37473/dac/10.1101/2020.09.25.20201459

20. Uyoga S, Adetifa IMO, Karanja HK, et al. Seroprevalence of anti-SARS-CoV-2 IgG antibodies in Kenyan blood donors. MedRxiv. https://doi.org/10.1101/2020.07.27.20162693

21. Agusi, E. R., Ijoma, S. I., Nnochin, C. S., Njoku-Achu, N. O., Nwosuh, C. I., \& Meseko, C. A. (2020). The COVID-19 pandemic and social distancing in Nigeria: ignorance or defiance. Pan African Medical Journal, 35(Supp 2). doi: 10.11604/pamj.supp.2020.35.2.23649

22. Mennechet, F., \& Dzomo, G. (2020). Coping with COVID-19 in Sub-Saharan Africa: What Might the Future Hold?. Virologica Sinica, 1-10. Advance online publication. https://doi.org/10.1007/s12250020-00279-2

23. Jin, J.-M., Bai, P., He, W., Wu, F., Liu, X.-F., Han, D.-M., ... Yang, J.-K. (2020). Gender differences in patients with COVID-19: Focus on severity and mortality. doi:10.1101/2020.02.23.20026864

24. Zhang JJ, Dong X, Cao YY, Yuan YD, Yang YB, Yan YQ, et al. Clinical characteristics of 140 patients infected by SARS-CoV-2 in Wuhan, China. Allergy. (2020). doi: 10.1111/all.14238. [Epub ahead of print].PubMed Abstract | CrossRef Full Text | Google Scholar

25. Wang D, Hu B, Hu C, Zhu F, Liu X, Zhang J, et al. Clinical characteristics of 138 hospitalized patients with 2019 novel coronavirus-infected pneumonia in Wuhan, China. JAMA. (2020). doi:

10.1001/jama.2020.1585. [Epub ahead of print]

26. Lee P-I, Hu Y-L, Chen P-Y, et al. Are children less susceptible to COVID-19? J Microbiol Immunol Infect 2020.

27. Altmann, D. M., Douek, D. C., \& Boyton, R. J. (2020). What policy makers need to know about COVID19 protective immunity. The Lancet, 395(10236), 1527-1529. doi:10.1016/s0140-6736(20)30985-5

28. Prado-Vivar, Belen and Becerra-Wong, Monica and Guadalupe, Juan Jose and Marquez, Sully and Gutierrez, Bernardo and Rojas-Silva, Patricio and Grunauer, Michelle and Trueba, Gabriel and Barragan, Veronica and Cardenas, Paul, COVID-19 Re-Infection by a Phylogenetically Distinct SARSCoV-2 Variant, First Confirmed Event in South America. (September 3, 2020). Available at SSRN: https://ssrn.com/abstract=3686174 or http://dx.doi.org/10.2139/ssrn.3686174

29. Jan Van Elslande, Pieter Vermeersch, Kris Vandervoort, Tony Wawina-Bokalanga, Bert Vanmechelen, Elke Wollants, Lies Laenen, Emmanuel André, Marc Van Ranst, Katrien Lagrou, Piet Maes, Symptomatic SARS-CoV-2 reinfection by a phylogenetically distinct strain, Clinical Infectious Diseases, , ciaa1330, https://doi.org/10.1093/cid/ciaa1330

30. Tillett RL, Sevinsky JR, Hartley PD et al. Genomic evidence for reinfection with SARS-CoV-2: a case study.Lancet Infect Dis. 2020; (published online Oct 12.)https://doi.org/10.1016/S1473- 
3099(20)30764-7

31. Kelvin Kai-Wang To, Ivan Fan-Ngai Hung, Jonathan Daniel Ip, Allen Wing-Ho Chu, Wan-Mui Chan, Anthony Raymond Tam, Carol Ho-Yan Fong, Shuofeng Yuan, Hoi-Wah Tsoi, Anthony Chin-Ki Ng, Larry Lap-Yip Lee, Polk Wan, Eugene Yuk-Keung Tso, Wing-Kin To, Dominic Ngai-Chong Tsang, KwokHung Chan, Jian-Dong Huang, Kin-Hang Kok, Vincent Chi-Chung Cheng, Kwok-Yung Yuen, Coronavirus Disease 2019 (COVID-19) Re-infection by a Phylogenetically Distinct Severe Acute Respiratory Syndrome Coronavirus 2 Strain Confirmed by Whole Genome Sequencing, Clinical Infectious Diseases, , ciaa1275, https://doi.org/10.1093/cid/ciaa1275

\section{Tables}

Table 1

Demographics of blood donors

\begin{tabular}{|lll|}
\hline Variable & Number & Percentage \\
\hline Sex & & \\
\hline Male & 106 & 94 \\
\hline Female & 7 & 6 \\
\hline Location & & \\
\hline Abuja & 67 & 59 \\
\hline Enugu & 46 & 41 \\
\hline Sample size & 113 & 100 \\
\hline
\end{tabular}


Table 2

Descriptive statistics of SARS CoV-2 IgG and IgM antibodies in blood donors

\begin{tabular}{|c|c|c|}
\hline Variable & SARS CoV-2 IgG & SARS CoV-2 IgM \\
\hline Median antibody level (NTU) & 19 & 15 \\
\hline (POSITIVE cases) & $(n=48)$ & $(n=44)$ \\
\hline $95 \% \mathrm{Cl}$ of Median & $17-21$ & 14-17 \\
\hline IQR antibody level (NTU) & 9 & 6 \\
\hline Median antibody level (NTU) & 5 & 6 \\
\hline (NEGATIVE cases) & $(n=46)$ & $(n=48)$ \\
\hline $95 \% \mathrm{Cl}$ of Median & $4-6$ & $5-7$ \\
\hline IQR antibody level (NTU) & 2 & 2 \\
\hline Median antibody level (NTU) & 10 & 10 \\
\hline (EQUIVOCAL cases) & $(n=12)$ & $(n=21)$ \\
\hline $95 \% \mathrm{Cl}$ of Median & $9-10$ & $9-10$ \\
\hline IQR antibody level (NTU) & 1 & 1 \\
\hline
\end{tabular}

Table 3

Inferential Statistics on SARS CoV-2 IgG and IgM antibodies in blood donors

\begin{tabular}{|lll|}
\hline Variable & P value for IgG & P value for IgM \\
\hline Positive vs. Negative cases & $<0.0001$ & $<0.0001$ \\
\hline Positive vs. Equivocal cases & 0.0071 & 0.0005 \\
\hline Negative vs. Equivocal cases & 0.0113 & 0.0002 \\
\hline $\begin{array}{l}\text { Kruskal wallis and Dunn's multiple comparison tests were performed. Kruskal wallis test showed } \\
\text { significant diferences in lgG and lgM antibodies for positive, negative and equivocal cases ( } p \text { value = } \\
\text { 0.0001 for lgG and } p \text { value =0.0001 for lgM). }\end{array}$ & \\
\hline
\end{tabular}

